Article

\title{
Optical Feedback Interferometry Based Microfluidic Sensing: Impact of Multi-Parameters on Doppler Spectral Properties
}

\author{
Yu Zhao ${ }^{1,2,3}$, Thierry Camps ${ }^{1}$, Véronique Bardinal ${ }^{1}$ and Julien Perchoux ${ }^{1, *(D)}$ \\ 1 LAAS-CNRS, Université de Toulouse, CNRS, INP, UPS, F-31400 Toulouse, France; \\ zhaoyuile@bjut.edu.cn (Y.Z.); thierry.camps@laas.fr (T.C.); bardinal@laas.fr (V.B.) \\ 2 Institute of Laser Engineering, Beijing University of Technology, Beijing 100124, China \\ 3 Beijing Engineering Research Center of Laser Technology, Beijing 100124, China \\ * Correspondence: perchoux@laas.fr; Tel.: +33-534-322-560
}

Received: 1 August 2019; Accepted: 20 August 2019; Published: 18 September 2019

\begin{abstract}
As a compact and simple sensing technique, optical feedback interferometry (OFI) can be a promising flowmetry method in various microfluidic applications. In this paper, OFI-based flowmetry sensor performance in a microscale flow scheme is studied theoretically and experimentally. An innovating model and different numerical methods are investigated, where the scattering light angle distribution is involved to predict the Doppler frequency distribution. For the first time, our model describes the influences of multiple OFI sensor system characteristics, such as flowing particle size, concentration, channel interface reflectivity and channel dimension, on the OFI signal spectral performances. In particular, a significant OFI signal level enhancement was achieved by deposing a high reflectivity gold layer on the rear channel interface due to the increased forward scattered light reflection. The consistent experimental validation associated with the simulations verifies this numerical simulation method's reliability. The numerical methods presented here provide a new tool to design novel microfluidic reactors and sensors.
\end{abstract}

Keywords: optical feedback interferometry; microfluidics; optical simulation; VCSELs; spectral imaging; sensing mechanisms

\section{Introduction}

In the last few decades, miniaturized and low reagent-consuming microfluidic devices have drawn great interest in biomedical and chemical engineering domains [1-3]. In microfluidic systems, the reagent flowing velocity inside a channel determines the reaction duration or the liquid mixing performance, therefore the flowing velocity or volume flow rate measurement is fundamental for microfluidic operation. Moreover, the flowmetry sensor in microfluidic chips is also of direct interest in biomedical and medical applications, e.g., assessment of burn depth, skin cancer diagnostics and extracorporeal blood flow monitoring [4-6]. Hence, numerous velocimetry technologies have been proposed in a microfluidic context [7-10].

Thanks to its various outstanding advantages such as low-cost, non-invasive, and self-alignment, optical feedback interferometry (OFI) offers a novel alternative for determining flow rate or velocity within flow-channels. Like traditional laser Doppler velocimetry (LDV) [11-13], this sensor measures the Doppler shift in the frequency spectrum of laser output power when it is subject to optical feedback from flowing particles. The spectrum is then an image of the velocity of the scatterers in the solution. A number of OFI-based flowmetry sensing research works have been reported [14-18]. However, these works were not evaluated as a systematic function of the particle characteristics and channel 
structure, which are essential in microfluidic sensing. Due to the lack of theoretical modeling, thus far, OFI-based microfluidic flowmeter sensor study remains challenging, especially with flowing channels of dimension smaller than $100 \mu \mathrm{m}$.

Involving different fundamental parameters, a synthetic numerical modeling approach using multiple simulation methods is demonstrated in this paper to predict the sensor signal behavior in the presence of optical feedback modulation in microfluidic sensing measurements. In order to validate our modeling method, a microfluidic flowmetry measurement setup was established, and a series of numerical simulations and flow rate measurements were performed based on this system. Comparisons of the simulation results and the measurement were investigated and discussed, then several important system factors influencing the OFI signal spectrum were highlighted; particle size, particle concentration, channel surface reflectivity and channel dimension.

\section{Materials and Methods}

\subsection{Theory}

When a part of the laser emission is reflected or scattered from an external target and then reinjected into the laser diode (LD) cavity, this feedback light provokes a fluctuation of the laser output power that carries information about the moving target. Using well-known three-mirror compound cavity models [19], the laser output power $P_{f}$ can be expressed as:

$$
\begin{aligned}
& P_{f}(t)=P_{0}\left[1+m \cdot \cos \left(2 \pi f_{d} t\right)\right] \\
& m=4 \xi \frac{\tau_{p}}{\tau_{l}}=2 \frac{r_{\text {ext }}\left(1-r_{2}^{2}\right) \tau_{p}}{r_{2} L}
\end{aligned}
$$

where $\lambda$ denotes the wavelength, $m$ is the feedback modulation factor determining the feedback strength, which depends on the laser output mirror reflectivity $r_{2}$, the external cavity length $L$, the photon lifetime $\tau_{p}$, and the feedback coupling efficiency $r_{e x t} . f_{d}$ is the Doppler frequency shift which is proportional to the scalar product of the vector difference between incidence and scattering beam vector and the velocity vector $\vec{V}$. The absolute value of $f_{d}$ can be expressed under the following form [20]:

$$
\left|f_{d}\right|=\frac{|\vec{k} \cdot \vec{V}|}{2 \pi}=\frac{V\left|\sin \theta_{\text {sca }}+\sin \theta_{\text {inc }}\right|}{\lambda}
$$

In usual flow measurements with OFI, the optical system collects the scattered light from a large number of particles (scatterers), and the dispersion of the scatterer velocity $\vec{V}$ and the scattering light direction $\theta_{s c a}$ induces a distribution of the Doppler frequency shifts $f_{d}$ that impact the LD output power fluctuation (in Figure 1). To evaluate the feedback output power behavior in the complicated microfluidic context, $P_{f}$ can be reconstructed as the sum of the feedback contributions from all particles in all the scattering directions:

$$
P_{f}(t)=P_{0}\left[1+\sum_{i} \sum_{k} m_{i} \cos \left(2 \pi f_{d i, k} t\right)\right]
$$

The attenuation of the liquid during beam propagation and the effect of the scatterer density on the scattering light power must also be taken into account here. The attenuated power after propagation distance $z$ can be derived by the usual extinction formula [21]:

$$
\begin{gathered}
P=P_{0} \exp (-\delta z) \\
\delta=N_{p} \cdot \sigma
\end{gathered}
$$

where $\delta$ is the extinction coefficient, $N_{p}$ is the particle density and $\sigma$ is the particle extinction cross-section. 


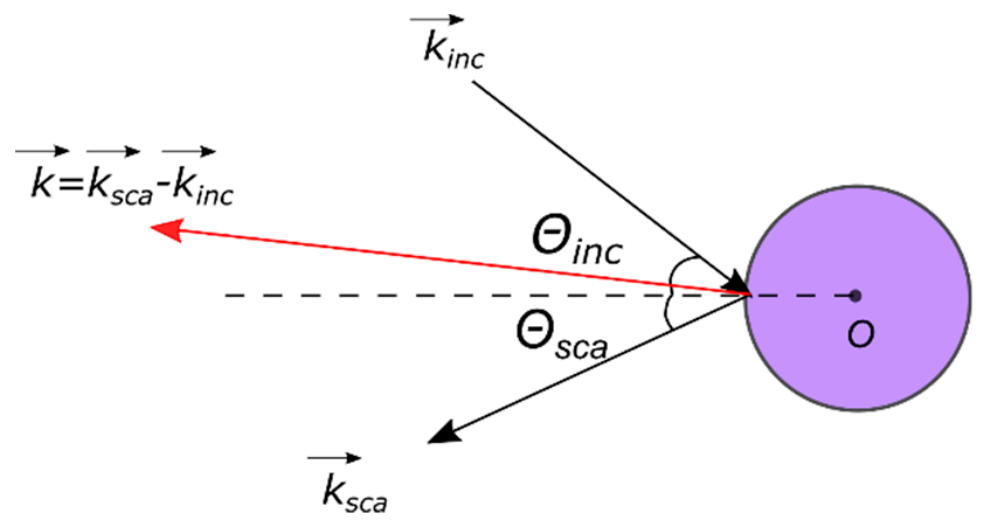

Figure 1. Incidence and scattering vectors when the laser beam was shot at the particle.

Considering that with higher particle density, more particles can be captured in the unit measurement period, the increase of density induces a higher OFI signal level. Thus, the particle density should also be added in the OFI signal expression. Finally, the OFI cosine function in Equation (4) can be corrected to reach the following complex form:

$$
P_{f}(t)=P_{0}\left[1+\sum_{i} \sum_{k} m_{i} N_{p} \exp \left(-2 \delta z_{i}\right) \cos \left(2 \pi f_{d i, k} t\right)\right]
$$

Because of the round-trip light propagation in the OFI system, the radiation is attenuated twice through the solution; thus, a factor of 2 has been added in Equation (7).

\subsection{Setup}

A microfluidic flowmetry system is built in this work for numerical simulation and experimental validation, the setup is illustrated in Figure 2a. One $1 \mathrm{~mm} \times 1 \mathrm{~mm}$ polydimethylsiloxane (PDMS) channel and two $96 \mu \mathrm{m} \times 96 \mu \mathrm{m}$ dry film (DF) photoresist channels realized the microfluidic reactors, and one of the DF channels was built with an additional gold coating film on the rear interface to demonstrate the channel reflectivity influence of the OFI signal. The OFI sensor is based on a $670 \mathrm{~nm}$ VCSEL (Vertical-Cavity Surface-Emitting Laser)at a constant bias current of $3.5 \mathrm{~mA}$ through a custom-designed laser driver. The incident angle $\theta$ between the laser emission axis $Z^{\prime}$ and perpendicular to the plane of the channel chip is set to $15^{\circ}$ as this value has experimentally proven the best signal level. A doublet lens pair (MAP103030B, Thorlabs Co., Ltd, Newton, NJ, USA) constituted by two lenses of the same focal length of $30 \mathrm{~mm}$ was used for laser beam focalization, resulting in a $4.6 \mu \mathrm{m}\left(1 / \mathrm{e}^{2}\right)$ laser beam waist in the micro-channel. Polystyrene (PS) microspheres (MicroParticles, $\mathrm{GmbH}$, Berlin, Germany) of two different diameters ( $552 \mathrm{~nm}$ and $4.96 \mu \mathrm{m}$ ) were used as the tracing particles. The PS solutions were pumped into the channel by a syringe pressing pump (PHD 22/2000 Harvard Apparatus, Holliston, MA, USA). The OFI signal was achieved through the monitor photodiode embedded in the VCSEL package. After amplification by a custom-made transimpedance amplifier, the OFI signal was acquired using a National Instrument acquisition card (NI USB 6251, National Instruments, Austin, TX, USA) at a sampling frequency of $400 \mathrm{kHz}$. Processing of the signal consists in averaging of 30 consecutive Fast Fourier Transform (FFT) of $2^{15}$ samples. All the data acquisition procedures and processing were automated by a custom-made LabVIEW algorithm. 


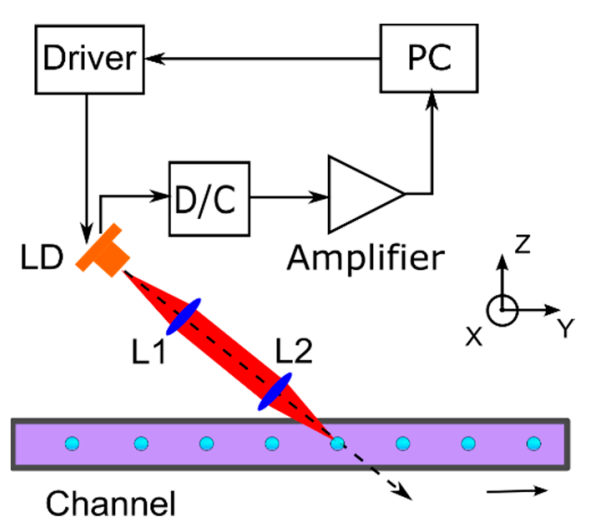

(a)

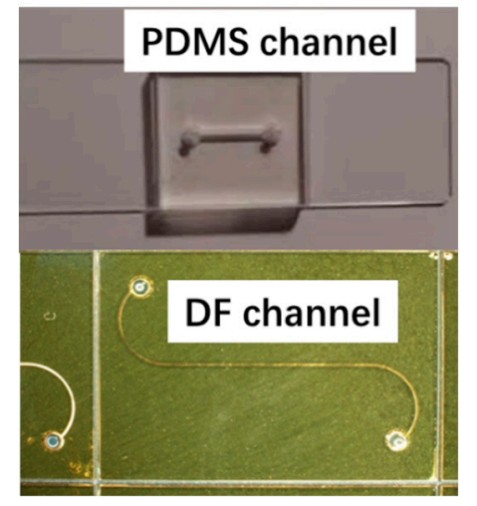

(b)

Figure 2. (a) Schematic of the microfluidic measurement system; (b) a polydimethylsiloxane (PDMS) channel and a dry film (DF) channel.

To implement our theoretical model, multiple numerical calculation tools were applied. Firstly, the entire optical part of the OFI system and the microfluidic channel chip were modeled using the commercial ray tracing software Zemax to simulate the laser beam propagation. All parameters such as channel geometrical sizes, materials, lens film coatings, particle characteristics were chosen to be in a realistic agreement with the experimental parameters in the 3D model. To calculate the OFI signal in different particle positions, a cuboid mesh was built inside the channel, and the fine $3 \mathrm{D}$ grid intervals were set to be $d x=d y=0.5 \mu \mathrm{m}, \mathrm{dz}=1 \mu \mathrm{m}$. A PS particle was placed at each node and then the ray tracing processing was repeated at all the mesh node coordinates. By the Monte Carlo statistical approach, the individual particle scattering angular distribution and the feedback light power re-entering-cavity coupling efficiency $r_{\text {ext }}$ were computed. Secondly, the flowing velocities at each node were calculated through simulation with computational fluid dynamics software Fluent. Finally, a Matlab algorithm has been designed that calculated the feedback modulation contributions to $P_{f}$ and the associated Doppler frequency shift $f_{d}$ as expected from Equations (3) and (7) and that simulates the Doppler frequency spectrum signal. The values of the modeling parameters are listed in Table 1.

Table 1. ZEMAX modeling parameters settings.

\begin{tabular}{cc}
\hline Simulation Parameters & Value \\
\hline Wavelength & $\lambda=670 \mathrm{~nm}$ \\
Half divergence of the laser & $B=5^{\circ}$ \\
Lens focal length & $f_{1}=f_{2}=30 \mathrm{~mm}$ \\
Refractive index of water & $n_{0}=1.33$ \\
Refractive index of PDMS & $n_{p}=1.4$ \\
Refractive index of DF & $n_{s}=1.59$ \\
Refractive index of PS particles & $N=1.58$ \\
Photon lifetime in laser & $\tau_{p}=10^{-12} \mathrm{~s}$ \\
External cavity length & $L=76 \mathrm{~mm}$ \\
VCSEL aperture diameter & $D A=6 \mu \mathrm{m}$ \\
Laser output cavity mirror & $r_{2}=0.9995$ \\
reflectivity & \\
\hline
\end{tabular}

\section{Results and Discussion}

Both simulations and experiments were applied here with the fluidic systems presented above to assay the OFI signal performance. In the following subsections, the results are demonstrated and discussed in detail to verify our model reliability. 


\subsection{Numerical Simulation Results}

Using the simulation method described above, the feedback contribution in different particle positions were assayed by calculating the irradiance distribution and the feedback light power re-entering-cavity coupling efficiency $r_{e x t}$ profile (sensing volume). Different parameters configurations were considered in this model to analyze the OFI sensor performance in different system schemes, and to emphasize the model availability in the practical applications.

Firstly, the OFI flowmetry sensor working in a macroscale reactor was investigated to understand the impact of channel dimension on the spectrum. The 2D incident profile and the feedback-power ratio $r_{\text {ext }}$ distribution through the macroscale PDMS channel (Figure 2b) are illustrated in Figure 3. In Figure 3a, one can observe that after the focalization with the lens pair, the entire laser focusing spot was only located in a small area (around $200 \mu \mathrm{m} \times 6 \mu \mathrm{m}$ ) close to the center of the channel. This laser illumination spatial limitation gave rise to an impressed coupling efficiency $r_{\text {ext }}$ distribution volume in a same dimension order (Figure 3b). Only the particles passing through a central area around $200 \mu \mathrm{m}$ can contribute efficiently to the feedback signal into the laser.

(a)

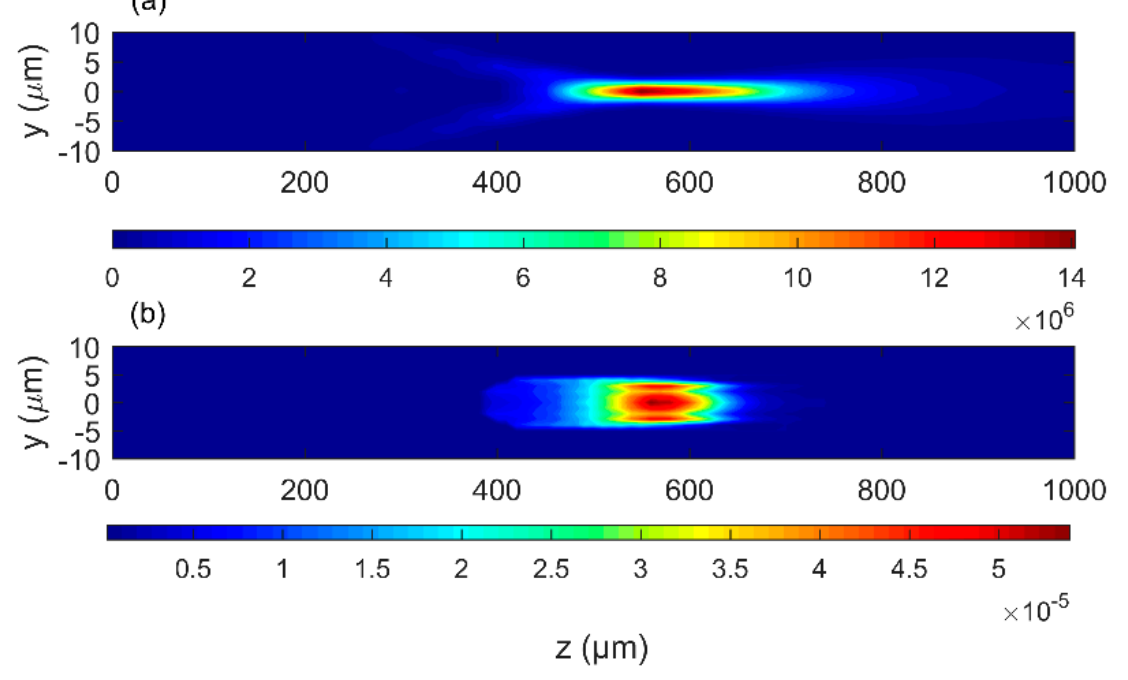

Figure 3. Two-dimensional simulation profile inside a $1 \mathrm{~mm} \times 1 \mathrm{~mm}$ PDMS channel. (a) Illumination power density profile; (b) feedback-power ratio profile.

The numerical simulations were repeated in the cases of the two microscale DF channels (Figure 4). In comparison to the case in macroscale channel, the feedback behavior in the microscale channel is more complicated. In Figure $4 \mathrm{a}$, the illumination profile here presents the same magnitude $\left(10^{6}\right)$. Contrary to the macroscale PDMS channel, due to the tiny channel dimension, only part of the laser spot can be coupled inside the microchannel in spite of the same tight focalization. Considering that the confocal parameter (twice the Rayleigh range) of the laser is approximatively $80 \mu \mathrm{m}$, comparable with the channel dimension $(96 \mu \mathrm{m})$, the laser beam $1 / \mathrm{e}^{2}$ radius inside the channel can be approximated to be constant at roughly $3 \mu \mathrm{m}$, which is represented as a parallel profile in Figure $4 \mathrm{a}$. 
(a)

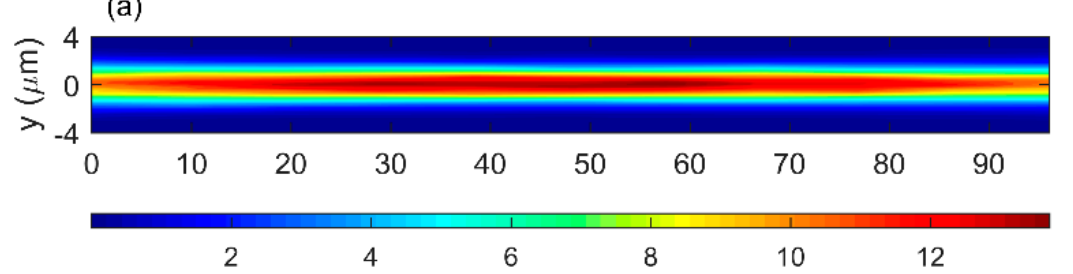

(b)

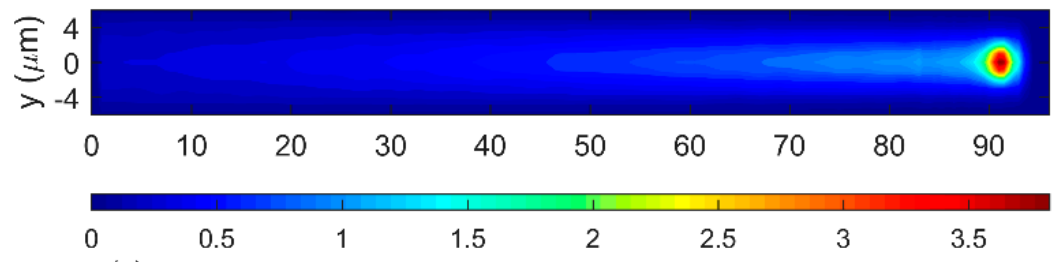

(c)

$\times 10^{-4}$

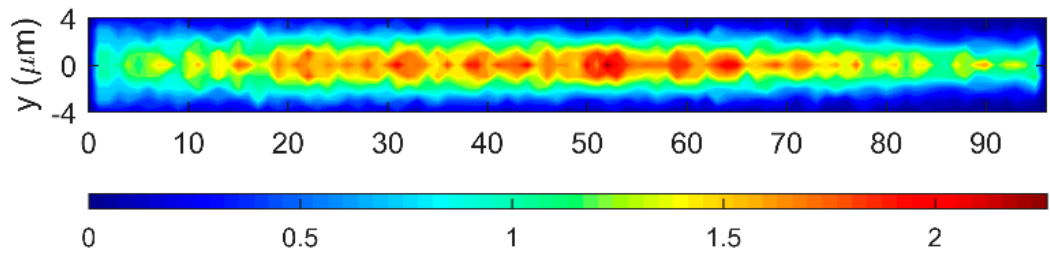

(d) $\times 10^{-6}$

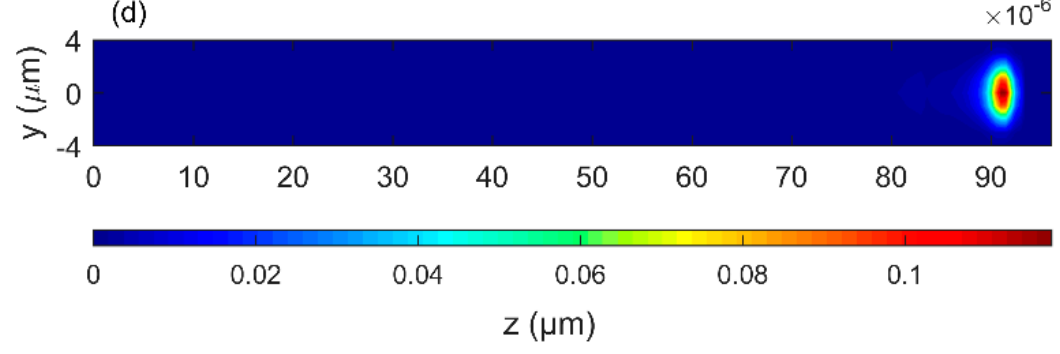

Figure 4. 2D simulation contour profiles in $96 \mu \mathrm{m} \times 96 \mu \mathrm{m}$ DF channel: (a) The illumination irradiance profile through the channel; (b) The feedback light power re-entering-cavity coupling efficiency $r_{\text {ext }}$ profile of $4.89 \mu \mathrm{m}$ PS particle in the channel without any coating; (c) $r_{\text {ext }}$ profile of $552 \mathrm{~nm}$ PS particle in the channel without gold coating; (d) $r_{\text {ext }}$ profile of $4.89 \mu \mathrm{m}$ PS in the channel with gold coating on the rear interface.

Then, the feedback light power re-entering-cavity coupling efficiency $r_{\text {ext }}$ in the channel where no high reflectivity gold coating was deposited on the rear interface is computed and its distribution is plotted in Figure $4 \mathrm{~b}$, where it exhibited a strong enhancement close to the rear interface. The probable explanation of this phenomenon can be the result of the forward scattered light that was focused by the particle and then reflected on the rear interface [22].

In order to evaluate the particle size effect on this phenomenon, we have changed the simulation parameter to a particle diameter of $552 \mathrm{~nm}$. The distribution of $r_{\text {ext }}$ in this condition is presented in Figure 4c. As compared with the distribution computed with $4.89 \mu \mathrm{m}$ PS particles, we did not observe any enhancement of $r_{\text {ext }}$ near the rear wall of the channel and the volume within which the particle scattering light can re-enter the cavity is comparable with the illumination profile. The maximum of $r_{\text {ext }}$ locates in the channel center, at the laser focal point. Because the scattered light power directly depends on the particle cross-section, the $r_{\text {ext }}$ value with the $552 \mathrm{~nm}$ PS particles were around 100 times lower than the one of $4.89 \mu \mathrm{m}$. On the other hand, such a small particle does not focus the forward scattered light efficiently onto the rear interface, which explains the absence of $r_{\text {ext }}$ enhancement.

At last, we model the case of the same size channel with a $50 \mathrm{~nm}$ thick additional high reflectivity gold film on the rear interface to increase the effect of the reflection of the forward scattering light. The coupling efficiency profile is plotted in Figure $4 \mathrm{~d}$ for $4.89 \mu \mathrm{m}$ particles. As expected a feedback enhancement point re-appears in the exact same position but this time, the coupling efficiency $r_{e x t}$ in the 
enhancement region is 300 times higher than in the non-coated channel of Figure $4 \mathrm{~b}$. These simulation results provide strong evidence for our mentioned hypothesis that the feedback light enhancement resulting from the forward scattering light reflection on the rear channel interface. Based on the coupling efficiency and parabolic particle velocity distribution in laminar fluid, one can simulate the OFI signal spectrum by Equations (3) and (7). A noise level is added in each simulated spectrum to make the results more realistic.

\subsection{Experimental Results}

In the previous subsection, the ray tracing simulation provided a solid understanding of the optical phenomena in the channel and the coupling coefficient $r_{\text {ext }}$ distribution. Here, a series of flowmetry measurements were performed for validation. Both simulated and measured OFI signal spectra are compared and investigated to analyze the influence of several important factors on the OFI signal spectra features: The particle size, the particle concentration, the reflectivity of the channel rear interface and the channel dimension (Figures 5-8).

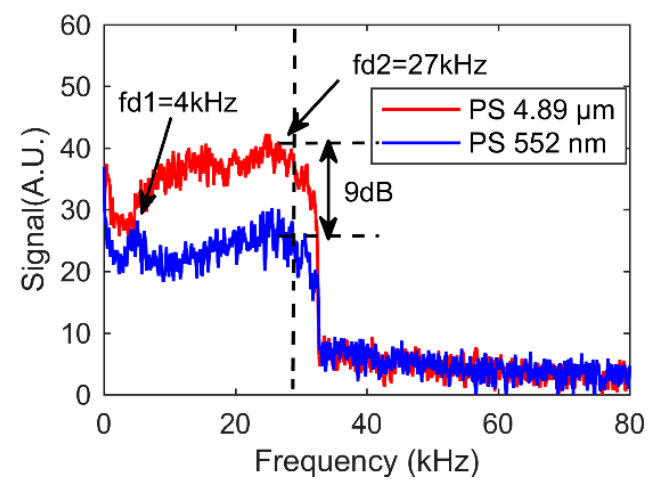

(a)

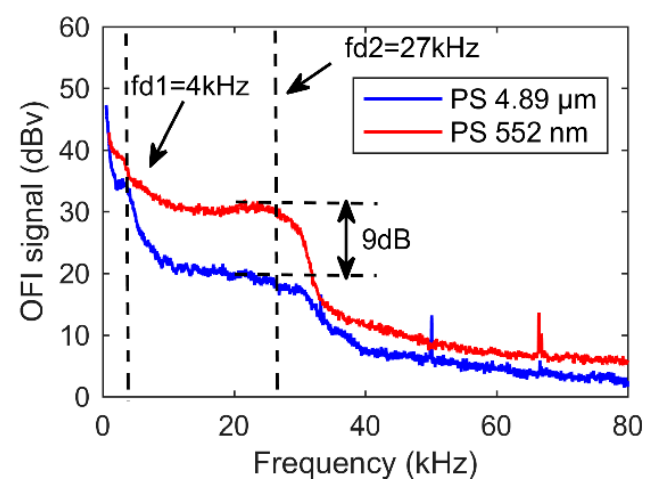

(b)

Figure 5. Optical feedback interferometry (OFI) signal frequency spectra obtained with PS particles of $552 \mathrm{~nm}$ diameter (red), $4.89 \mu \mathrm{m}$ (blue): (a) simulated spectra; (b) measured spectra.

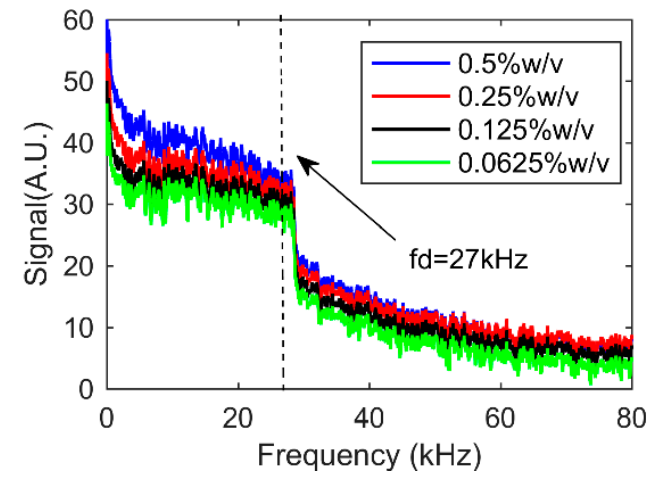

(a)

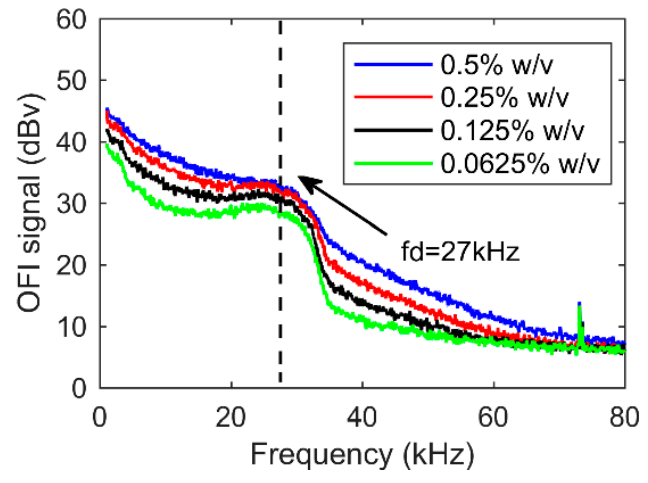

(b)

Figure 6. OFI signal frequency spectra obtained in $552 \mathrm{~nm}$ PS particle solution at concentration of $0.625 \% w / v$ (green), $0.125 \% w / v$ (black), $0.25 \% w / v$ (red), $0.5 \% w / v$ (blue): (a) simulated spectra; (b) measured spectra. 


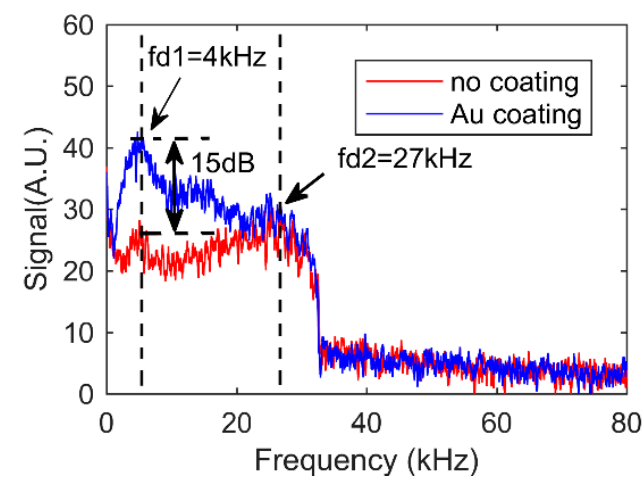

(a)

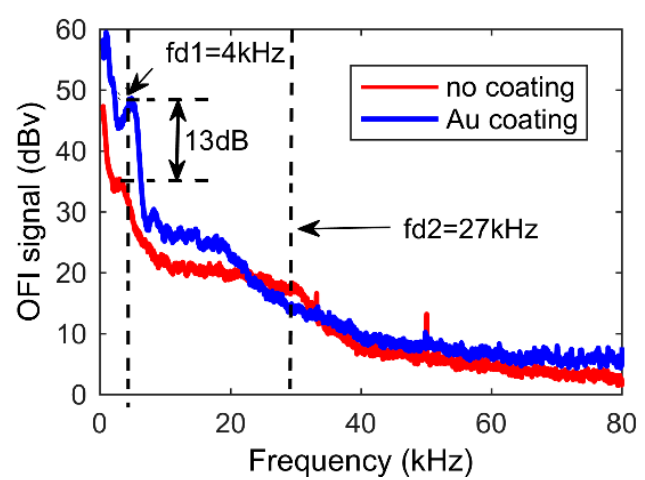

(b)

Figure 7. The OFI signal frequency spectra obtained in a $96 \mu \mathrm{m} \times 96 \mu \mathrm{m}$ DF channel without coating (red) and with a gold coating on the rear interface (blue): (a) simulated spectra; (b) measured spectra.

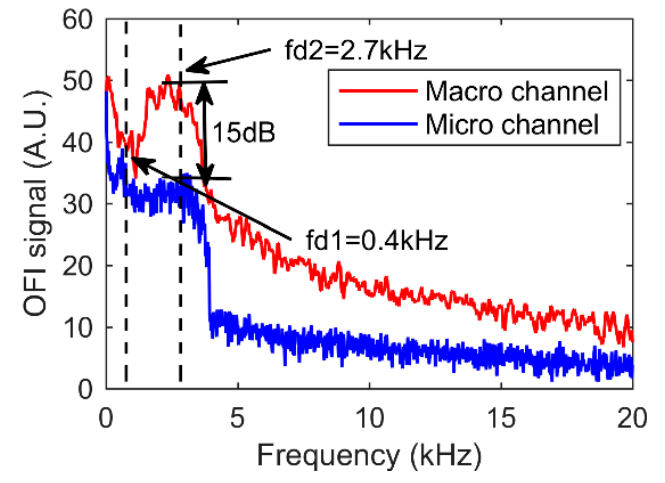

(a)

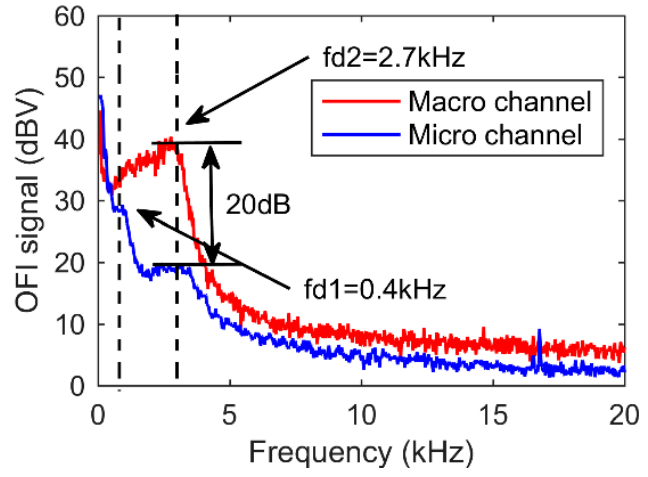

(b)

Figure 8. The OFI signal frequency spectra obtained with PS particles of PDMS macro channel at $\mathrm{Q}=100 \mu \mathrm{L} / \mathrm{min}$ (red), DF micro channel $\mathrm{Q}=1 \mu \mathrm{L} / \mathrm{min}$ (blue): (a) simulated spectra; (b) measured spectra.

\subsubsection{Particle Size}

Firstly, the influence of particle size on the OFI signal was investigated. The OFI microfluidic flowmetry simulations and measurements with $552 \mathrm{~nm}$ and $4.89 \mu \mathrm{m}$ diameter PS particles at the same concentration of $0.1 \% w / v$ and flow rate of $\mathrm{Q}=10 \mu \mathrm{L} / \mathrm{min}$ are compared in Figure 5a (simulations) and Figure $5 \mathrm{~b}$ (measurements). For the larger particle (blue solid lines in both figures), a low frequency peak $f d 1$ corresponding to the forward scattered light reflection induced feedback enhancement appears, whereas the spectra of the smaller PS particle (red solid line) does not present such frequency peak. The $f d 1$ value is calculated to be around $4 \mathrm{kHz}$ considering the velocity distribution within the enhancement area at $\mathrm{Q}=10 \mu \mathrm{L} / \mathrm{min}$. It is also interesting to note that the $552 \mathrm{~nm}$ particles produce a spectral signal level that is $9 \mathrm{~dB}$ higher than the $4.89 \mu \mathrm{m}$ particles at the frequency $f d 2$ that correspond to the theoretical maximum Doppler shift. The signal level difference can be explained by the larger particle density (number in unit volume). Since the concentrations in weight per volume are the same, so that in the $4.89 \mu \mathrm{m}$ particles solution, the particle density is around 1000 times less than for $552 \mathrm{~nm}$ particles. As can be seen both in the measurement and in the simulation, the larger scattering cross-section of $4.89 \mu \mathrm{m}$ particles does not compensate the lower number of particles contributing to back-scattered light.

\subsubsection{Particle Concentration}

Various concentrations, expressed in weight per volume $(w / v)$, of $552 \mathrm{~nm}$ PS particle solutions $(0.0625 \% w / v, 0.125 \% w / v, 0.25 \% w / v, 0.5 \% w / v)$ have been tested, always with a flow rate $\mathrm{Q}=10 \mu \mathrm{L} / \mathrm{min}$. The resulting simulated and measured spectra are plotted in Figure $6 \mathrm{a}, \mathrm{b}$, respectively. From the simulation results shown in Figure 6a, one can observe that the spectral signal level remains alike 
over the whole particle concentration range in particular at the expected maximum Doppler shift $f d=27 \mathrm{kHz}$, while in the low frequency region $(<20 \mathrm{kHz})$, the increase in concentrations enhanced slightly the signal level. This behavior can be explained that when the particle density increases, the laser beam cannot enter deeper in the channel, and thus only the particles passing through the top area of the channel with a lower flowing velocity can properly contribute to the optical feedback, resulting in such a slight signal increase in the low frequency region.

\subsubsection{Rear Interface Reflectivity}

Eventually, the OFI signal simulations and measurements are performed in the DF channels with and without the additional gold coating on the rear channel interface. The simulated OFI signal spectra in the two channels are plotted (red-without coating and blue-with coating) in Figure 7a while measured ones are plotted with the same color code in Figure $7 \mathrm{~b}$. As can be seen, simulated and measured spectra have clearly a different behavior in the low frequencies as the measurement exhibits a regular increase of the power at low frequencies. We believe this effect to be the result of unwanted vibrations induced fringes that are emphasized considering the higher reflectivity of the gold coating. However, a similar trend can be observed in the measured and simulated OFI signals: Both spectra have a visible lower frequency peak $f d 1=4 \mathrm{kHz}$ that corresponds to the reflection induced feedback-power enhancement as depicted in Figure $4 \mathrm{~b}$,d. Moreover, the magnitude difference between the peak at $f d 1$ obtained with the gold coating and the one observed without coating is of $15 \mathrm{~dB}$ in the simulated spectra and $13 \mathrm{~dB}$ higher in the measured ones, indicating a consistent agreement, proving the essential role of the rear interface reflectivity.

\subsubsection{Channel Dimension}

At last, OFI sensor behavior dependence on the fluidic channel dimension was considered as well. In Figure 8, we performed the simulation and measurement of an OFI signal spectra in $1 \mathrm{~mm} \times 1 \mathrm{~mm}$ macroscale PDMS at $100 \mu \mathrm{L} / \mathrm{min}$ flow rate and $96 \mu \mathrm{m} \times 96 \mu \mathrm{m}$ microscale DF channel at $1 \mu \mathrm{L} / \mathrm{min}$, respectively. The 10 times flow rate difference was applied to maintain the similar velocity and Doppler frequency in the cases of different channel cross-section dimension.

In the simulation of the macro PDMS channel (red in Figure 8a), the relatively small sensing volume with respect to the entire channel size (shown in Figure 3b) led in a sharp Doppler frequency peak at $f d 2=2.7 \mathrm{kHz}$ in the frequency spectrum. Considering the entire laser spot is located inside the channel, almost all the laser emission power can be scattered by the flowing particles, the simulated spectra exhibited a high signal level. However, in the micro DF channel, the comparable sensing volume with the entire channel dimension in Figure $4 \mathrm{~b}$ shows that all the particles can contribute the OFI signal of different Doppler frequency shift segments, giving rise to a frequency plateau (blue in Figure 8a). Considering only part of the emission is coupled in the microchannel, the resulting spectra was $15 \mathrm{~dB}$ lower. Again, a secondary frequency peak $f d 1$ appears in DF channel due to the reflection enhancement. In Figure 8b, the measured spectra in both channels exhibit the semblable appearances at $f d 2=2.7 \mathrm{kHz}$, sharp peak in the macro channel and plateau in the microchannel, denoting the maximal velocity at the center where the laser focal point was positioned, and the secondary peak $f d 1=0.4 \mathrm{kHz}$ was only in DF channel. The signal difference was $20 \mathrm{~dB}$ between the two channels, indicating a consistent agreement with the simulation.

\section{Conclusions}

In this paper, a new numerical simulation approach of the OFI sensing scheme was developed that simulated macro/microfluidic flowmetry sensor signal spectra while taking into account the largest number of experimental configuration parameters. The laser beam propagation through the complex microscale fluidic system was simulated by a ray tracing method. The feedback-power coupling efficiency distribution simulation was emphasized here to understand the sensing volume of the system in different system configurations. Different important parameters have been tested to validate 
the modeling approach: The particle size, density, the reflectivity of the rear flow-channel walls and the channel dimension. The measurement produced the signal that is consistent with the simulated ones, thus successfully proving the capability of our numerical method. The series of measurements in all the parameter configurations were also repeated at different flow rates, presenting a promising capacity of the sensor system as well. Such a sensor behavior model could be a major asset while designing micro-reactors with dedicated optical properties. This model can further be extended to consider other parameters, such as the nature of the particle or the one of the carrying fluids. Thus, considering the extreme compactness of OFI scheme, this method is paving the way towards embedded flowmeters to be integrated into lab-on-a-chip devices.

Author Contributions: The contribution of the authors to the realization of this article is detailed as follows: Software simulation methodology, experimental validation, visualization, investigation and writing, Y.Z.; reviewing, T.C.; supervision, V.B.; conceptualization, J.P.

Funding: This research was funded by Beijing municipal Natural Science Foundation, Grant Number 4194071 and the open project of Beijing Engineering Researching Center of Laser Technology, Grant Number BG0046-2018-xx.

Acknowledgments: The authors gratefully acknowledge Véronique Conédéra, Fabien Mesnilgrente and Rémi Courson for their contribution in DF microfluidic channel fabrication processing. The authors also sincerely appreciate Clement Tronche and Francis Jayat for their help in electronic designing work.

Conflicts of Interest: The authors declare no conflict of interest.

\section{References}

1. Whitesides, G.M. The origins and the future of microfluidics. Nature 2006, 442, 368-373. [CrossRef] [PubMed]

2. Velve-Casquillas, G.; Le Berre, M.; Piel, M.; Tran, P.T. Microfluidic tools for cell biological research. Nano Today 2010, 5, 28-47. [CrossRef] [PubMed]

3. Khandurina, J.; Guttman, A. Bioanalysis in microfluidic devices. J. Chromatogr. A 2002, 943, $159-183$. [CrossRef]

4. Vennemann, P.; Lindken, R.; Westerweel, J. In vivo whole-field blood velocity measurement techniques. Exp. Fluids 2007, 42, 495-511. [CrossRef]

5. Draijer, M.; Hondebrink, E.; Van Leeuwen, T.; Steenbergen, W. Review of laser speckle contrast techniques for visualizing tissue perfusion. Lasers Med. Sci. 2009, 24, 639-651. [CrossRef] [PubMed]

6. Chen, Z.; Milner, T.E.; Dave, D.; Nelson, J.S. Optical Doppler tomographic imaging of fluid flow velocity in highly scattering media. Opt. Lett. 1997, 22, 64-66. [CrossRef] [PubMed]

7. Abbiss, J.B. Principles and practice of laser-Doppler anemometry. Opt. Laser Technol. 1976, 8, $236-237$. [CrossRef]

8. König, J.; Voigt, A.; Büttner, L.; Czarske, J. Precise micro flow rate measurements by a laser Doppler velocity profile sensor with time division multiplexing. Meas. Sci. Technol. 2010, 21, 074005-074014.

9. Pape, S.A.; Skouras, C.A.; Byrne, P.O. An audit of the use of laser Doppler imaging (LDI) in the assessment of burns of intermediate depth. Burns 2001, 27, 233-239. [CrossRef]

10. Adrian, R.J. Twenty years of particle image velocimetry. Exp. Fluids 2005, 39, 159-169. [CrossRef]

11. Mazumder, M.K. Laser doppler velocity measurement without directional ambiguity by using frequency shifted incident beams. Appl. Phys. Lett. 1970, 16, 462-464. [CrossRef]

12. Buttner, L.; Czarske, J.; Knuppertz, H. Laser-Doppler velocity profile sensor with submicrometer spatial resolution that employs fiber optics and a diffractive lens. Appl. Opt. 2005, 44, 2274-2280. [CrossRef] [PubMed]

13. Oliveira, R.; Semedo, S.; Figueiras, E.; Ferreira, L.F.R.; Humeau, A. Laser Doppler flowmeters for microcirculation measurements. In Proceedings of the 1st Portuguese Meeting in Bioengineering 2011, Lisbon, Portugal, 1-4 March 2011; pp. 1-4.

14. Rovati, L.; Cattini, S.; Palanisamy, N. Measurement of the fluid-velocity profile using a self-mixing superluminescent diode. Meas. Sci. Technol. 2011, 22, 025402-025411. [CrossRef]

15. Campagnolo, L.; Nikolić, M.; Perchoux, J.; Lim, Y.; Bertling, K.; Loubière, K.; Prat, L.; Rakić, A.D.; Bosch, T. Flow profile measurement in microchannel using the optical feedback interferometry sensing technique. Microfluid. Nanofluidics 2013, 14, 113-119. [CrossRef] 
16. Lim, Y.; Nikolic, M.; Bertling, K.; Kliese, R.; Rakic, A.D. Self-mixing imaging sensor using a monolithic VCSEL array with parallel readout. Opt. Express 2009, 17, 5517-5525. [CrossRef] [PubMed]

17. Ramírez-Miquet, E.E.; Perchoux, J.; Loubière, K.; Tronche, C.; Prat, L.; Sotolongo-Costa, O. Optical feedback interferometry for velocity measurement of parallel liquid-liquid flows in a microchannel. Sensors 2016, 16, 1223-1235. [CrossRef] [PubMed]

18. Mowla, A.; Taimre, T.; Lim, Y.; Bertling, K.; Wilson, S.J.; Prow, T.W.; Rakić, A.D. A Compact Laser Imaging System for Concurrent Reflectance Confocal Microscopy and Laser Doppler Flowmetry. IEEE Photonics J. 2016, 8, 1-9. [CrossRef]

19. Wang, W.M.; V Grattan, K.T.; Palmer, A.W.; Boyle, W.J.O. Self-mixing interference inside a single-mode diode laser for optical sensing applications. J. Lightwave Technol. 1994, 12, 1577-1587. [CrossRef]

20. Zhao, Y.; Perchoux, J.; Camps, T.; Bardinal, V. Optical Feedback Interferometry Flowmetry Sensor in Microfluidics. In Proceedings of the Eurosensors 2017, Paris, France, 3-6 September 2017; pp. 507-511.

21. Hecht, E. Optics, 4th ed.; Addison Wesley Longman Inc.: Boston, MA, USA, 1998; pp. 131-135.

22. Zhao, Y.; Perchoux, J.; Campagnolo, L.; Camps, T.; Atashkhooei, R.; Bardinal, V. Optical feedback interferometry for microscale-flow sensing study: Numerical simulation and experimental validation. Opt. Express 2016, 24, 23849-23861. [CrossRef] [PubMed]

(C) 2019 by the authors. Licensee MDPI, Basel, Switzerland. This article is an open access article distributed under the terms and conditions of the Creative Commons Attribution (CC BY) license (http://creativecommons.org/licenses/by/4.0/). 